\title{
Trypsine et bactérie entéropathogène : d'un commun accord pour activer la production des prostaglandines intestinales!
}

\begin{abstract}
Alors que les prostaglandines règlent de multiples fonctions intestinales telles que le transport ionique, la croissance cellulaire, le flux sanguin, et jouent le rôle de médiateurs de l'inflammation et de la cytoprotection, les événements qui contrôlent la synthèse et la sécrétion de ces agents par l'intestin lui-même restent quasiment inexplorés à ce jour. Dans ce contexte, deux articles récents démontrant le rôle activateur respectivement de la trypsine et des bactéries invasives entéropathogènes sur la production des icosanoïdes intestinales constituent des informations essentielles pour la compréhension des mécanismes moléculaires impliqués dans la physiologie et la physiopathologie intestinale [1, 2].
\end{abstract}

Dans la première étude, la trypsine, enzyme digestive d'origine pancréatique, par son action stimulante sur la production de prostaglandines intestinales, apparaît comme une molécule de signalisation importante dans le contrôle paracrine et autocrine de la cellule cible entérocytaire [1]. Déjà décrite comme proche de la thrombine par son mécanisme d'action sur un tissu cible [3], la trypsine agirait par clivage et activation de récepteurs PAR (proteinase-activated receptor) appartenant à une nouvelle famille de récepteurs couplés aux protéines $G$, et ayant pour ligands des protéases à serine. En effet, le récepteur de la trypsine, désigné PAR-2, possède dans son domaine extra cellulaire un site de clivage spécifique par l'enzyme, à côté duquel existe une séquence correspondant au vrai ligand du récepteur capable d'activer le récepteur clivé. Ayant déjà décrit l'expression des récepteurs PAR-2 dans la sphère digestive, le rein, l'œil et l'ovaire [4], les auteurs mettent aujourd'hui clairement en évidence l'importance du rôle physiologique de la trypsine dans la régulation de l'épithélium intestinal, et notamment la mise en jeu de la synthèse de prostaglandines. L'étude d'une lignée de cellules intestinales épithéliales de rat en culture (lignée hBRIE) et des muqueuses de duodénum, jéjunum, iléon et côlon de rat a permis d'identifier des récepteurs PAR-2 à la fois dans les villosités et les cryptes et, plus précisément, dans les membranes basolatérales et apicales des entérocytes, au niveau de l'appareil de Golgi et dans des vésicules. Les cellules hBRIE activées par la trypsine ou par le peptide correspondant au ligand du récepteur PAR-2 de rat (désigné AP) montrent une augmentation de la production d'inositol trisphosphate (InsP3) intracellulaire (en $10 \mathrm{sec}$ ), suivie d'une augmentation de la production d'acide arachidonique (précurseur des prostaglandines) et de la sécrétion des prostaglandines PGE2 et PGF1 $\alpha$ (entre 5 et 15 minutes). Enfin, dans un modèle d'intestin de rat éversé, l'application de trypsine ou du peptide AP au niveau de la muqueuse jéjunale induit une forte stimulation de la sécrétion de PGE2 en moins de 30 minutes. Ainsi, outre son rôle dans la digestion, la trypsine luminale, par la libération de prostaglandines intestinales, peut être désormais considérée comme une molécule de signalisation importante des cellules entérocytaires.

Une situation pathogénique comme l'infection intestinale par des bactéries invasives semble également conduire à la mobilisation des icosa- noïdes du tractus digestif. C'est la conclusion d'une étude anglo-américaine qui démontre que, dans les cellules intestinales épithéliales infectées par les bactéries entéropathogènes du genre Salmonella, l'augmentation de la sécrétion hydroélectrolytique qui suit l'infection est relayée par les prostaglandines, mettant en jeu directement une enzyme clé responsable de leur synthèse, la PGHS (prostaglandine H synthase) [2]. Les bactéries, du genre Salmonella sont responsables de maladies fréquentes chez l'homme (dysenterie, gastroentérite), et ont un taux de mortalité important dans les pays en voie de développement [5]. L'implication des prostaglandines dans la réponse de l'hôte à l'infection bactérienne a été suggérée après l'observation que l'infection orale de singes par Salmonella induit une stimulation rapide de la sécrétion hydroélectrolytique par un mécanisme sensible à l'indométhacine, un inhibiteur de la PGHS. Cette hypothèse vient d'être confirmée lors de l'infection de lignées intestinales épithéliales humaines (lignée 1407, HT29) par différentes bactéries invasives du genre Salmonella. En effet, des cellules mises en contact une heure avec la bactérie $S$. dublin voient leur capacité de sécréter les prostaglandines PGE2 et PGF2 $\alpha$ augmentée d'un facteur 50, effet bloqué spécifiquement par l'indométhacine. La production de ces prostaglandines dépend de la concentration de l'inoculum bactérien; observée dès 3 heures après l'infection bactérienne, elle atteint un plateau en 12 heures. Des deux isoformes PGHS-1 et PGHS-2 existantes, PGHS-2 semble la plus impliquée, comme en témoigne l'augmen- 
tation du transcrit du gène PGHS-2, et de l'enzyme dans les cellules infectées, augmentation parallèle à celle des prostaglandines sécrétées. Alors que seules les bactéries invasives sont actives, une relation directe entre leur entrée dans la cellule et l'expression de PGHS-2 a pu être définitivement établie au cours des 5 premières heures qui suivent l'infection. In vivo, dans un modèle de xénogreffe d'intestin humain chez la souris, l'infection de la greffe par une bactérie invasive conduit à une augmentation de plus de 40 fois de la synthèse des transcrits $P G H S-2$. Enfin, fait confirmé à l'aide d'un anticorps anti-PGE2 ajouté au milieu, les PGE2 sécrétées par HT29 sont capables d'induire spécifiquement une augmentation de la sécrétion de chlorure par des cellules de côlon polarisées en culture, et représentent donc le secrétagogue dominant produit par la cellule intestinale épithéliale après infection bactérienne. Dans cette situation physiopathologique, les prostaglandines ainsi produites joueraient-elles également un rôle protecteur sur la muqueuse intestinale (effet mitogénique, antiapoptotique, sécrétion de mucus)?

\section{B.A.}

1. Kong W, McConalogue K, Khitin LM, Hollenberg MD, Payan DG, Böhm SK, Bunnett NW. Luminal trypsin may regulate enterocytes through proteinase-activated receptor 2. Proc Natl Acad Sci USA 1997 ; 94 : 8884-9.

2. Eckmann L, Stenson WS, Savidge TC, Lowe DC, Barrett KE, Fierer J, Smith JR, Kagnoff MF. Role of intestinal epithelial cells in the host secretory response to infection by invasive bacteria. Bacterial entry induces epithelial prostaglandin $\mathrm{H}$ synthase-2 expression and prostaglandin E2 and F2 $\alpha$ production. J Clin Invest 1997; $100: 296-309$. 3. Van Obberghen-Shilling E, Pouysségur. Le récepteur de la thrombine et ses implications dans la prolifération des cellules vasculaires. Med Sci 1993 ; 9 : 1043-9.

4. Böhm SK, Kong W, Brömme D, Smeekens SP, Anderson DC, Connolly A, Kahn M, Nelken NA, Coughlin SR, Payan DG, Bunnett NW. Molecular cloning, expression and potential functions of the human proteinase-activated receptor-2. Biochem J $1996 ; 314: 1009-16$

5. Ménard R, Sansonetti P. Signaux moléculaires induisant l'entrée des bactéries entéropathogènes dans les cellules épithéliales : convergences et paradoxes. Med Sci 1996 ; 12 : 465-73.
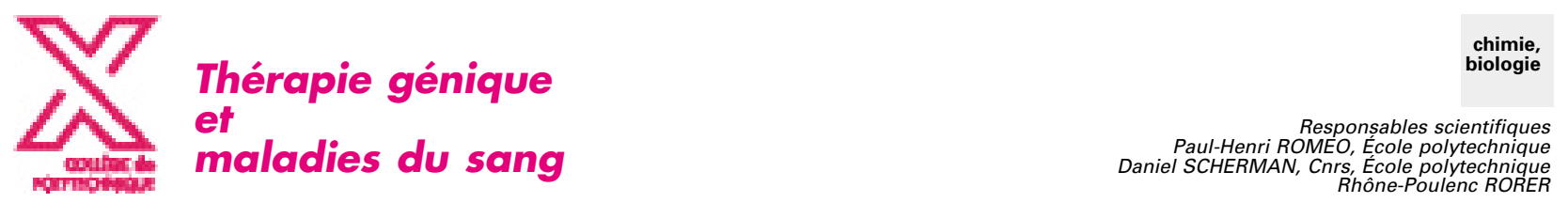

Le transfert de gènes dans des cellules humaines a pu être envisagé, lorsque les mécanismes moléculaires de l'induction de certains cancers par des virus ont été découverts. Ce transfert semble être une des voies les plus prometteuses dans le traitement des maladies génétiques et des maladies acquises comme le Sida, les cancers solides, ou les leucémies.

De plus, le renouvellement extrêmement dynamique des cellules du sang, via les cellules hématopoïétiques, est souvent compromis lors des chimiothérapies lourdes employées dans le traitement de certains cancers. Dans ce cas, la thérapie génique permet de rendre ces cellules résistantes à la chimiothérapie.

Les progrès dans les domaines de la biologie cellulaire des cellules hématopoiétiques, de l'étude moléculaire de l'expression des gènes spécifiques de ces cellules, et du transfert de gènes annoncent des révolutions thérapeutiques majeures.

Intervenants

Patrice DENEFLE, Directeur de Recherches, Division Thérapie Génique et Cellulaire, RHÔNE POULENC RORER

Anne DUBART, Institut Gustave-Roussy

Paul-Henri ROMEO, Maître de Conférences à l'École polytechnique, Directeur de I'Unité Génétique Moléculaire et Hématologie, Inserm

Daniel SCHERMAN, Directeur de Recherches Cnrs, Maître de Conférence à l'École polytechnique, Directeur de l'unité mixte Cnrs - Rhône Poulenc Rorer "vectorologie moléculaire"

Frédéric TRIEBEL, Professeur, Directeur de I'Unité Système Immunitaire et Cancers, Inserm

William VAINCHENKER, Directeur de Recherches, Directeur de I'Unité Hématopoïèse et Cellules Souches, Inserm

Programme

Introduction à la thérapie génique

- Transfert de gènes dans les cellules somatiques

- Maladies susceptibles de bénéficier d'une thérapie génique

Biologie cellulaire de I'hématopoïèse

- Les cellules souches hématopoiétiques

- Régulation de la production des cellules différenciées par les facteurs de croissance

- La thérapie génique du système hématopoḯtique est-elle possible ?

Biologie moléculaire de l'hématopoï̀se

- Du gène à la protéine : les mécanismes de l'expression génétique - Les différentes catégories de cellules sanguines expriment des gènes différents
Objectifs

- exposer les bases cellulaires et moléculaires du transfert de gènes en général, dans les cellules hématopoiétiques en particulier

- présenter les différentes stratégies de modification du patrimoine génétique de ces cellules

- montrer les bénéfices que la santé humaine peut trouver dans le développement de ces nouvelles technologies

Public

Médecins, pharmaciens, ingénieurs, cadres et chercheurs en chimie, pharmacologie et biologie

- Comment assurer l'expression d'un gène correcteur?

Thérapie génique et immunologie

- Les acteurs de la réponse immunitaire chez l'individu sain

- Le traitement des déficits immunitaires primitifs par thérapie génique

- Les modificateurs de la réponse biologique et leur emploi dans les maladies acquises du système immunitaire: maladies autoimmunes, cancers, Sida.

Quels vecteurs pour la thérapie génique ?

- Vecteurs viraux et non viraux : leurs avantages spécifiques

- Du transfert de gène expérimental à la réalité clinique, les étapes à franchir

- Les vecteurs de demain
1 jour
16 janvier 1998
École polytechnique, Palaiseau $3000 \mathrm{~F} \mathrm{HT}$ 